\title{
ASUNTOS CONCEPTUALES, METODOLÓGICOS Y ÉTICOS DE LA HIPNOSIS COMO TERAPIA PSICOLÓGICA ADJUNTA AL TRATAMIENTO DEL CÁNCER DE MAMA
}

CONCEPTUAL, METHODOLOGICAL AND ETHICAL ISSUES SURROUNDING HYPNOSIS WHEN USED AS PSYCHOLOGICAL THERAPY ATTACHED TO BREAST CANCER TREATMENT

DOI: 10.22199/S07187475.2012.0002.00007

Recibido: 13 de Junio del 2012 | Aceptado: 20 de Agosto del 2012

JULIO SANTANA MARIÑ $0_{1}$; GUILLERMO BERNAL 2 LYDIA RODRIGUEZ 2 ; KEISHALEE GÓMEZ 2 ; JENNIFER

MORALES,

(UNIVERSIDAD DE PUERTO RICO Recinto Río Piedras, San Juan, Puerto Rico).

\begin{abstract}
RESUMEN
La hipnosis ha formado parte de intervenciones psicológicas para atender los efectos de múltiples condiciones oncológicas y sus tratamientos. En este trabajo revisamos los principales resultados de la investigación existente en donde se utiliza la hipnosis en pacientes con cáncer de mama. En la primera parte se discuten asuntos conceptuales y metodológicos, en la segunda parte los hallazgos, limitaciones y asuntos éticos de la hipnosis en el debate de las prácticas psicológicas. En los estudios revisados se evidencia consistentemente que la hipnosis es eficaz para tratar el dolor, síntomas de ansiedad, sofocos (hot flashes), angustia previa a la biopsia de mama y efectos secundarios de la quimioterapia y radioterapia. El documento concluye con sugerencias para investigaciones futuras y las implicaciones para la práctica clínica y reconoce a la hipnosis como un recurso valioso para mejorar el estado de ánimo y la calidad de vida las pacientes de cáncer de mama. PALABRAS CLAVE: Cáncer de mama, Hipnosis, Asuntos metodológicos y conceptuales.
\end{abstract}

\section{ABSTRACT}

Hypnosis is one of a number of psychological interventions which can be used to address the effects of multiple oncological conditions and treatments thereof. In this paper we review existing research results regarding the use of hypnosis in patients with breast cancer. In the first part we discuss conceptual and methodological issues. In the second part we discuss the findings, limitations and ethical issues of hypnosis in the on-going debate surrounding psychological practices. Consistent evidence exists in the reviewed research studies that hypnosis is effective in treating pain, anxiety symptoms, hot flashes, pre-breast biopsy anxiety, and the side effects of chemotherapy and radiotherapy. We conclude with suggestions for future research, implications for clinical practice, and the recognition of hypnosis as a valuable resource for improving both mood and quality of life of breast cancer patients.

KEY WORDS: Breast cancer, Hypnosis, Conceptual and methodological issues. 
INTRODUCCIÓN

El cáncer constituye uno de los mayores problemas que enfrenta el sector de la salud pública en países desarrollados, particularmente en los Estados Unidos de América (EUA), donde actualmente una de cada cuatro muertes se debe a esa enfermedad. El Centro Nacional de Cáncer de ese país estimó que en el 2009 se diagnosticaron 192,370 mujeres con cáncer de mama y aproximadamente 40,170 morirían por esta condición (Jemal, et al., 2009). En Puerto Rico, el cáncer de mama es el tipo de cáncer más diagnosticado en las mujeres y representa el $33.0 \%$ de todos los tipos de cáncer diagnosticados en las mujeres durante el periodo de 1999 - 2003. Este cáncer es la primera causa de muerte por cáncer en las mujeres y representa el $17.8 \%$ de todas las muertes por cáncer en las mujeres durante el periodo de 2000 al 2004 en Puerto Rico (Registro Central de Cáncer de Puerto Rico, 2008).

Abundante literatura describe la sintomatología psicológica y los significados que tiene para las pacientes de cáncer de mama el impacto de esta condición y sus tratamientos. La hipnosis ha formado parte de intervenciones para atender los efectos de múltiples condiciones oncológicas. En este trabajo nos proponemos revisar los principales resultados de la investigación existente en donde se utiliza la hipnosis en pacientes con cáncer de mama. En la primera parte se discutirán asuntos conceptuales y metodológicos, en la segunda parte los hallazgos y retos fundamentales de estos estudios. En la Tabla 1 se resumen estos estudios a partir de las variables de interés en este trabajo.

\section{Intervención con hipnosis y cáncer de mama}

La larga y prolífica producción científica sobre la eficacia de los tratamientos conductuales contrasta con la exigua divulgación de las intervenciones con hipnosis en pacientes diagnosticadas con cáncer de mama. La hipnosis tiene una larga trayectoria integrada al tratamiento de múltiples condiciones de salud física y mental. En los últimos 15 años se han realizado rigurosas revisiones y metaanálisis donde se describe el impacto de la intervención con hipnosis para controlar dolor, nauseas y vómitos como efectos secundarios de la quimioterapia, mejorar calidad de vida de pacientes y la respuesta autoinmune en adultos y niños con cáncer. Sin embargo, no existe revisión sobre la utilización de la hipnosis en pacientes con cáncer de mama (Liossi, 2006; Mendoza \& Capafons, 2009; Montgomery \& Schnur, 2005; Richardson, et al., 2007). Después de revisar los principales meta-análisis y revisiones de hipnosis aplicada al cáncer, fue sorprendente encontrar solo 11 trabajos publicados en idioma inglés que dieron cuenta del uso de la hipnosis en pacientes con cáncer de mama. Se destacan 7ensayos clínicos aleatorizados, dos ensayos clínicos no aleatorizados y dos reportes de caso (Elkins, Jensen, \& Patterson, 2007; Montgomery, David, Winkel, Silverstein, \& Bovbjerg, 2002). A continuación realizaremos un análisis de la dimensión conceptual de la hipnosis en los estudios revisados.

\section{Asuntos conceptuales de la hipnosis}

En las diferentes revisiones sobre la investigación en hipnosis se trata someramente las maneras definir a la hipnosis. En los tiempos fundacionales se le llamó hipnosis, en franca alusión al sueño, pasando luego por mesmerismo, magnetismo animal, sueño lúcido, hipnoterapia, hipnotismo, sugestión, auto hipnosis y autosugestión. En tiempos más recientes se le ha llamado estado alterado de conciencia, disociación, visualización, relajación e imaginería (Kirsch, Lynn, \& Rhue, 1993). Cabe preguntarse si tantas definiciones tienen un impacto en la comprensión y justa valoración de este procedimiento. Este asunto conceptual tiene implicaciones teóricas y metodológicas que impactan el reconocimiento, aceptación 
o rechazo de los profesionales de salud y en última instancia, de las personas beneficiarias de este tratamiento. Una confusión sobre el proceso que llamamos hipnosis pondría en tela de juicio los resultados de eficacia cuando se comparan procesos diferentes.

En las investigaciones de hipnosis en cáncer de mama encontramos solo tres estudios que incluyeron una definición operativa de la hipnosis. El estudio llevado a cabo por Spiegel y Boom (1983) constituye la referencia de la eficacia de la hipnosis en pacientes con cáncer de mama. En él se define a la hipnosis como un proceso de focalización de la atención y se sugiere que cada persona puede aprender a usar esta capacidad de cambiar el foco de la atención y absorberse en la experiencia de las instrucciones de uno mismo. En otro estudio se utilizó la hipnosis como adyuvante de la terapia cognitiva conductual para tratar la claustrofobia en dos pacientes que recibirían radioterapia y se refiere a la hipnosis como un proceso de relajación instruyendo a las participantes a realizar auto hipnosis (Steggles, 1999). Por su parte Marchioro y colaboradores (2000) desarrollaron una intervención donde combinaron Terapia Cognitiva-Conductual (TCC) con técnicas de relajación y de hipnosis para tratar las náuseas y vómitos anticipatorios a la quimioterapia. En este trabajo no se ofrece una definición de hipnosis, pero describen los pasos de la inducción que realizó.

En otro estudio llevado a cabo por Montgomery y colaboradores (2002) para disminuir la angustia y estrés de un grupo de mujeres previo a la cirugía de mama, tampoco se define conceptualmente la hipnosis. En el estudio se hace referencia al procedimiento desarrollado por Kirsh y colaboradores (1993) y describe algunas de las instrucciones que contenía la inducción hipnótica donde destaca el uso del "lugar especial".
Elkins y colaboradores(2007; 2008) realizaron varias investigaciones con pacientes sobrevivientes de cáncer de mama para disminuir los hot flashes (sofocos) utilizando hipnosis Estos autores no definen a la hipnosis, sino que describen algunas instrucciones y mencionan instrucciones de frío y relajación. En otros dos trabajos llevados a cabo por Schnur y colaboradores (2009; 2008) para evaluar una intervención de Terapia Cognitiva Conductual combinada con Hipnosis en pacientes que recibieron tratamiento de radioterapia, no se plantea una definición operativa de la hipnosis, remitiéndose al procedimiento desarrollado por Kirsh (1993) y mencionando varias instrucciones del proceso de hipnosis.

Butler y colaboradores (2009) desarrollaron un estudio donde se incorpora la hipnosis al final de una intervención grupal de apoyo expresivo para disminuir el estrés y el dolor en un grupo de pacientes. En este trabajo aparece una clara definición operacional de la hipnosis y del procedimiento seguido. "La hipnosis es el estado de focalización de la atención donde se reduce la atención periferal del ambiente y aumenta la capacidad de respuesta a las señales sociales" (p.882).

En un trabajo reciente sobre la expectativa de respuesta como mediador de la hipnosis en pacientes después de la cirugía de mama, Montgomery y colaboradores (2010) definen la hipnosis del siguiente modo: "es un acuerdo entre una persona designada como hipnotista (profesional de la salud) y una persona designada como participante o cliente que participa de esta técnica psicoterapéutica, donde se sugieren cambios en la sensibilidad, percepción y la cognición, lo que afecta el estado de ánimo y el comportamiento" (p.80).

Al analizar la presencia de una definición de hipnosis en los principales investigaciones sobre cáncer de mama 
encontramos que solo tres de los estudios definen operativamente a la hipnosis, existiendo una diferencia fundamental en lo planteado por Spiegel y Butler con respecto a Montgomery, donde los primeros reducen la hipnosis al proceso de focalización de la atención que ocurre en la persona, mientras que este último ofrece una visión más amplia de la hipnosis, entendiéndola como un proceso de interacción entre paciente y terapeuta con el propósito de generar cambios en múltiples niveles de la persona.

Indudablemente este aspecto, por sus implicaciones tiene gran relevancia para la investigación. Aunque siempre van a existir diferentes maneras de abordar 0 describir las terapias psicológicas, es necesario esclarecer a qué nos referimos cuando decimos hipnosis en los estudios (a la disociación, la focalización de la atención, a la relajación, la visualización y cuáles componentes lo distinguen). En un loable esfuerzo por lograr un acercamiento en la histórica controversia sobre la definición de hipnosis la División 30 (Hipnosis Clínica) de la Asociación Americana de Psicología (APA), propuso una definición consensuada que, en sentido general, ha sido aceptada por las más importantes sociedades de hipnosis científica. Esta plantea que la hipnosis es un procedimiento mediante el cual se sugiere a las personas cambios en las sensaciones, percepciones, pensamientos, emociones o conducta. Esta División destaca que generalmente, el procedimiento hipnótico se inicia a través de un método de inducción que puede adoptar formas muy diferentes, aunque las más frecuentes son las que incluyen sugestiones de calma, relajación y bienestar, así como instrucciones de pensar 0 imaginar experiencias agradables. Son sugerencias para guiar al participante a relajarse, 0 concentrarse en pensamientos 0 imágenes. Los métodos de inducción son muy creativos y diversos, van a depender de los objetivos clínicos o de investigación y de los profesionales que la utilicen (APA, 1994; Green, Barabasz, Barrett, \& Montgomery,
2005; Kirsch, et al., 1993). Los planteamientos de la APA no cierran la discusión teórica y conceptual sobre la hipnosis pero constituyen un punto relevante en el debate y reconocimiento de este procedimiento en el campo de la investigación. A continuación se discutirán algunos asuntos metodológicos de gran importancia de la investigación realizada en el campo de la hipnosis aplicada al cáncer de mama.

\section{Análisis comparativo de la muestra}

En todos los estudios revisados, los pacientes fueron remitidos por oncólogos siendo todas las participantes provenientes de muestras clínicas (Butler, et al., 2009; Elkins, Marcus, et al., 2007; Elkins, et al., 2008; Marchioro, et al., 2000; Montgomery, et al., 2007; Montgomery, Weltz, et al., 2002; Spiegel \& Bloom, 1983; Steggles, 1999). Con respecto al tipo de tratamiento médico prevalecieron las pacientes que estaban próximas a recibir 0 recibieron radioterapia, seguidas por las que recibieron quimioterapia. También se desarrollaron con pacientes sobrevivientes de cáncer y otros estudios con pacientes que esperaban por cirugías y biopsias para diagnosticar cáncer de mama. En cuanto a grupos raciales, las participantes que prevalecieron en estos estudios fueron denominadas como blancas, representado más del $50 \%$ de los casos, del total integrado por afroamericanas, hispanas, asiáticas y caribeñas en uno de los estudios. Aunque no se establecen diferencias significativas atendiendo al estatus civil de las participantes, las mujeres que respondieron que estaban casadas, respresentan más del $50 \%$ de las que participaron en estos estudios.

Al comparar el tamaño de las muestras de todos estos estudios nos encontramos que las muestras oscilaron entre medianas y pequeñas. Montgomery y colaboradores (2007) desarrollaron una intervencion de hipnosis previa a la cirugía de cáncer de 
mama con doscientas participantes $(n=200)$, la cual constituye la mayor muestra para un estudio donde se ha utilizado la hipnosis. Se comparó un grupo que recibió una sesión de hipnosis de 10 minutos de duración previo a relizarse biopsias excisionales, con otro grupo que recibió la atención estándar. Los resultados de este estudio confirman que la hipnosis en ambientes clínicos puede modificar la expectativa de respuesta de los pacientes al dolor y disminuir la experiencia del dolor después de la cirugía. No se encontró diferencia significativa entre ambos grupos en cuanto a la prevención de náuseas después de la cirugía. En este caso se usó la hipnosis exclusivamente.

Dos estudios con muestras pequeñas fueron estudios de caso. El primero fue una intervención donde se utilizó la hipnosis previa a la sesiones de radioterapia en dos pacientes con cáncer de mama con historial de ansiedad fóbica, más especificamente fobia a los espacios cerrados (Steggles, 1999). Se decribe que una de las pacientes mantuvo niveles clínicos de ansiedad pero logró completar el tratamiento radiológico. El segundo estudio de caso, fue llevado a cabo recientemente. En este se comparó un tratamiento que combinó Terapia Cogntivo Conductual más hipnosis (TCCH) en el curso completo del tratamiento de radioterapia, con la intención de mejorar la fatiga y disminuir las incomodidades en la piel (2008). Se comparó el TCCH con el tratamiento estándar. En la paciente que recibió $\mathrm{TCCH}$ los niveles de fatiga fueron menores que la paciente control. Se mantuvieron bajos los niveles de angustia en la paciente que recibió TCCH y se incrementaron en la paciente con el tratamiento estándar duarante el curso de la radioterapia.

La mayoría de las participantes fueron seleccionadas después de haber sido diagnosticadas con cáncer. Se destacan los estudios llevados a cabo por Spiegel y Boom (1983) y Butler y colaboradores
(2009) donde el criterio de inclusión fue el diagnóstico de cáncer de mama metastásico. La severidad de los síntomas que provoca el cáncer y la mortalidad estadística por fallecimiento de las pacientes es un asunto a tener en cuenta en las intervenciones. En otros estudios la condición de metástasis fue considerada un criterio de exclusión (Schnur, et al., 2009)

Principales variables estudiadas en la investigación sobre hipnosis y cáncer de mama: dolor, ansiedad y sofocos

\section{Dolor}

Los reportes más amplios sobre la eficacia de la hipnosis están centrados en el manejo del dolor (Elkins, Jensen, et al., 2007). El estudio realizado por Spiegel y Bloom (1983) fue pionero en demostrar la eficacia de la hipnosis en el manejo del dolor en 56 mujeres con cáncer de mama (carcinoma metastásico) que participaron en una terapia de grupo a la cual se agregó hipnosis. La percepción y la frecuencia e intensidad de dolor, fueron las variables principales del estudio, aunque también se evaluaron los disturbios emocionales, la ansiedad, depresión y fatiga en el curso de un año utilizando cuatro puntos de evaluación cada cuatro meses. Los instrumentos empleados fueron una Escala de Rango de Dolor (SRD) (Spiegel \& Bloom, 1983) con una puntuación de 1-10, donde se les pidió a las participantes que calificaran la sensación del dolor y explicaran el sufrimiento asociado a la experiencia. Usaron la Escala Perfil de Estados de Ánimo (POMS) (Shacham, 1983) en su versión larga para evaluar los síntomas de ansiedad, fatiga y depresión.

Por su parte, Montgomery y sus colegas (2007) desarrollaron una investigación con 200 participantes, de las cuales 102 recibieron tratamiento de hipnosis previo a la cirugía de cáncer de mama y midieron la intensidad del dolor, incomodidad, náusea, 
fatiga, y malestar emocional postquirúrgico. Para medir estas variables utilizaron una escala visual análoga con rango de 1-100 (Montgomery, Weltz, et al., 2002; Spiegel \& Bloom, 1983), la cual fue presentada a las participantes al momento abandonar la hospitalización. Midieron, además el uso de sedantes y analgésicos empleados en estas pacientes y calcularon la reducción en costo por cada paciente para la institución.

Butler (2009) en una investigación más reciente, utilizó la hipnosis como adjunto a una terapia de apoyo expresivo, utilizando como variables el dolor, el sufrimiento, la frecuencia del dolor y el grado constante del dolor. Estas variables fueron medidas, al inicio y en cuatro períodos durante un año, utilizando la Escala de Rango de Dolor (Spiegel \& Bloom, 1983), y un cuestionario de retroalimentación.

En los tres estudios con pacientes de cáncer de mama es consistente la evaluación subjetiva del dolor en la apreciación de intensidad y frecuencia, así como en la experiencia de sufrimiento relacionada con el dolor. En estos estudios se destaca la relación del dolor con expresiones de ansiedad y depresión.

\section{Ansiedad}

Otra de las variables estudiadas en las investigaciones de cáncer de mama e hipnosis es la ansiedad. Esta variable ha sido utilizada bajo diferentes denominaciones: estrés, angustia, sufrimiento y más recientemente ubicada dentro del constructo de afecto negativo. Schnur (2009) evaluó cómo una intervención con TCCH disminuía el afecto negativo y aumentaba el positivo en un grupo de pacientes que recibieron radioterapia. Se trabajó con las variables rasgo ansiedad y rasgo de neuroticismo. Se utilizó la Subescala del Inventario de Neuroticismo de Cinco Factores (NEO-FFI) versión corta (Costa \& McCrae, 1992) y el Inventario de Ansiedad Rasgo Estado
(STAI) (Spielberger, 1983). La ansiedad fue medida usando la escala de tensión ansiedad del Perfil de Estados de Ánimo, versión corta (POMS-SV) (Baker, Denniston, Zabora, Polland, \& Dudley, 2002; Shacham, 1983). Este último instrumento ha sido el más usado para medir ansiedad en las intervenciones con hipnosis y cáncer de mama, al parecer por su confiabilidad y facilidad para contestarlo.

\section{Sofocos (hot flashes)}

Elkins y colaboradores $(2007 ; 2008)$ han desarrollado un trabajo destacado utilizando la variable sofocos (hot flashes) en pacientes sobrevivientes de cáncer de mama. Un sofoco es una sensación subjetiva de calor que está asociada con la vasodilatación y puede ser acompañado por sudoración, palpitaciones, ansiedad, irritabilidad, e incluso pánico. Este síntoma ha sido asociado a la menopausia provocada por la quimioterapia. Por sus implicaciones en la calidad de vida de las pacientes, estos autores han desarrollado varias intervenciones donde emplean la hipnosis para manejar estos síntomas. Los instrumentos empleados han sido los siguientes: Escala de Interferencia Diaria Relacionada a los Socofos (Hot Flash Related Daily Interference Scale) (Carpenter, 2001), para medir la frecuencia, intensidad y la manera en que interfieren en diferentes áreas de su vida cotidiana y la Sub escala de Ansiedad de la Escala de Ansiedad Hospitalaria (Hospital Anxiety and Depression Scale) (Zigmond \& Snaith, 1983); Escala de estudios médicos de sueño (Medical Outcomes Study Sleep) (Stewart \& Ware, 1992), fueron usadas respectivamente para medir el impacto de los sofocos en el sueño y en el estado de ánimo.

En sentido general, prevalecieron los autoinformes como instrumentos de evaluación de las variables, en la mayoría de las veces POMS y escalas visuales análogas. Las variables de depresión, 
náuseas, fatiga e irritabilidad de la piel han sido estudiadas con menos frecuencia. La fatiga fue estudiada inicialmente por Spiegel y más recientemente Montgomery y Schnur , pero entendemos que merce ser atendida en futuros trabajos, tomando en cuenta la frecuencia con que está siendo resportada por población con cáncer de mama.

Hallazgos fundamentales de la investigación con hipnosis y cáncer de mama

Luego de la exhaustiva revisión de la investigación en el tema que nos ocupa, salta a la vista un renovado interés por la utilización de la hipnosis en el curso del diagnóstico y tratamiento del cáncer de mama. Desde la década de los años 80 hasta el año 2000 solo identificamos tres investigaciones (Redd \& Andrykowski, 1982; Spiegel \& Bloom, 1983; Steggles, 1999). E triple de investigaciones en este tema se ha reportado en los últimos 10 años (Butler, et al., 2009; Elkins, Marcus, et al., 2007; Elkins, et al., 2008; Marchioro, et al., 2000; Montgomery, et al., 2007; Montgomery, Weltz, et al., 2002; Schnur, et al., 2009; Schnur \& Montgomery, 2008).

Las intervenciones en las que se incluyó la hipnosis para el control del dolor fueron más eficaces que aquellas donde se usaron estrategias de control de atención y educación. En el trabajo de Spiegel y colaboradores (1983) se reportó menos dolor y sufrimiento en las participantes que practicaron auto hipnosis; de manera similar Montgomery y colaboradores (2007) encontraron que las pacientes que pertenecian al grupo de hipnosis manifestaron menos intensidad del dolor e incomodidad que las que solo recibieron el tratamiento usual. En esa misma dirección Butler y colaboradores (2009) reportaron que la terapia de grupo mediante apoyo expresivo más la hipnosis, disminuyó la experiencia y el incremento del dolor con respecto al grupo que recibió solo educación.
Fue también eficaz la combinación de hipnosis con terapia cognitiva conductual para disminuir el afecto negativo y aumentar el bienestar psicológico (Schnur, et al., 2009), de igual modo la intervención con hipnosis fue eficaz para disminuir la frecuencia de sofocos (hot flashes) en un $68 \%$ cuando se comparan con un placebo (Elkins, et al., 2008). Estos resultados alentadores engrosan la evidencia de la utilidad clínica de la hipnosis como adyuvante de los tratamientos de pacientes con condiciones médicas. En dos de los estudios se evaluó la satisfacción de las participantes con el uso de la hipnosis; en ambos se informaron valoraciones favorables y en ninguno de los dos estudios las pacientes mencionaron efectos secundarios (Butler, et al., 2009; Elkins, et al., 2008).

Otro resultado importante fue identificar que en más del $50 \%$ de los estudios, la hipnosis fue integrada a otro tratamiento psicológico como terapias grupales $\mathrm{e}$ individuales, siendo combinada mayoritariamente con tratamientos cognitivos conductuales. Esto concuerda con la tendencia que reconoce la importancia de desarrollar tratamientos multimodales que complementen sinérgicamente su eficacia (Schnur, et al., 2009).

En sentido general, aunque son pocos los trabajos publicados, resulta alentador observar que más del $50 \%$ de las investigaciones con hipnosis en cáncer de mama son ensayos clínicos aleatorizados, lo que plantea el alto nivel de rigurosidad con que se está tratando el tema.

Limitaciones de los estudios con hipnosis y cáncer de mama

Aunque resulta alentador el incremento de evidencia generada por 11 estudios revisados en pacientes con cáncer de mama, pueden señalarse algunas debilidades básicas que se dan en el diseño 
de la mayoría de los informes. En los 11 estudios revisados en pacientes con cáncer de mama, las muestras pequeñas constituyen un problema que dificulta generalizar resultados y profundizar en los mecanismos que explican los cambios de la hipnosis. El tamaño pequeño de las muestras es una crítica consistente identificada en los meta-análisis y revisiones sobre hipnosis (Elkins, Jensen, et al., 2007). Los estudios carecen de grupo de comparación, lo que abre el cuestionamiento de si los resultados son un efecto de la intervención. Las evaluaciones de seguimiento se emplean a corto plazo, lo que dificulta determinar el efecto de las intervenciones a largo plazo.

De la revisión realizada sobresale la necesidad de una clara conceptualización de la hipnosis y por ende, de precisar los componentes de las intervenciones que utilizan la hipnosis, para comparar mejor los estudios y para diferenciar la hipnosis de otras intervenciones etiquetadas muchas veces con este nombre, como el entrenamiento en relajación. Sería de utilidad poder disponer de protocolos que describan las etapas, componentes y sugestiones específicas para generalizar su incorporación a los programas de tratamiento de los profesionales de la psicología clínica.

Desde el punto de vista sociodemográfico, las intervenciones se han desarrollado en mujeres denominadas tradicionalmente blancas residentes en Estados Unidos. Incluso en uno de los estudios sus autores reconocen que las participantes de su investigación pertenecían a clases socioeconómicamente altas, lo que limitaba la generalización de su intervención a personas pertenecientes a minorías étnicas, con otro trasfondo socioeconómico (Butler, et al., 2009).

También es una limitación consistente en las investigaciones estudiadas, la dificultad para explicar los mecanismos de cambios de las intervenciones con hipnosis. La excepción es el trabajo de Montgomery (2010) que sugiere el efecto moderador de la expectativa de respuesta y el estrés anticipatorio en la respuesta de dolor postquirúrgico. Aunque dos estudios revisados reportan un tamaño grande del efecto explicable por la hipnosis, un metaanálisis resulta necesario con esta población.

La hipnosis en el debate ético de las prácticas psicológicas

La hipnosis ha sido un procedimiento controversial desde su incorporación como herramienta terapéutica. Gran parte de estas controversias están relacionadas con múltiples mitos generalizados entre la población y algunos círculos profesionales. Trabajar o investigar en este campo supone discutir y eliminar mitos alimentados por las malas prácticas y por la televisión o el cine. Consideramos esta una responsabilidad ética que contribuye a un adecuado acercamiento de parte de los profesionales de la salud y de las personas beneficiarias. Hay que reconocer que en la mayoría de estudios recientes, previo a la intervención con hipnosis se discuten los temores y se aclaran las dudas de las participantes de la intervención.

El movimiento de prácticas basadas en evidencia puso de relieve un importante cuestionamiento ético a la hora de ofrecer los procedimientos psicológicos por los profesionales. ¿Se ofrece a clientes 0 pacientes la mejor evidencia disponible de terapias psicológicas para atender o tratar sus condiciones médicas 0 de salud mental? ¿Se ofrece el tratamiento o terapia que ha mostrado mayor evidencia de eficacia? ¿Las escuelas de psicología incluyen el adiestramiento en hipnosis basándose en los criterios de las prácticas basadas en evidencia? ¿La investigación en hipnosis se desarrolla tomando en cuenta estos criterios? 
Estos cuestionamientos de carácter ético tienen total pertinencia en el caso de la hipnosis. La eficacia de la hipnosis para manejar la ansiedad, el dolor, las náuseas, el estrés previo a la cirugía de cáncer de mama y durante el curso de la quimioterapia y radioterapia, está muy bien documentada desde hace más de 30 años (Montgomery \& Schnur, 2005; Spiegel \& Bloom, 1983). Sin embargo, no siempre forma parte del ofrecimiento terapéutico a nivel público 0 privado.

Tampoco los currículos de formación de médicos, enfermeros/as y psiquiatras y psicólogos/as clínicos tienen incluida la capacitación en hipnosis. No creemos que deba atenderse como un asunto de vocación personal, sino desde el punto de vista ético como una responsabilidad profesional. En una entrevista realizada a Kirsh, éste mencionó que en los Estados Unidos, se ofrecen cursos completos de hipnosis en aproximadamente el $25 \%$ de los programas de doctorado de Psicología Clínica. Un 14\% adicional ofrece cursos más amplios que incluyen instrucción en hipnosis. En el Reino Unido, aproximadamente el $45 \%$ de los departamentos de psicología enseña hipnosis; en Australia el panorama es del $75 \%$. Aclaró este autor que no tenía información sobre las escuelas de enfermería y odontología (Capafons, 1995). En Puerto Rico no existe información precisa pero conocemos que la mayor capacitación proviene de esfuerzos de asociaciones profesionales y grupos privados.

En la totalidad de los estudios de cáncer de mama los psicólogos/as clínicos refieren tener capacitación en hipnosis clínica. Elkins (2008) sugiere el adiestramiento más allá de un curso básico como un requisito para intervenir con esta población. Este aspecto supone un posicionamiento ético ineludible si tomamos en cuenta que ni en Estados Unidos, ni en Puerto Rico existe reglamentación sobre la práctica de la hipnosis. En algunos países como España, Inglaterra y Cuba la práctica con pacientes está limitada a profesionales de la salud y sus respectivos estudiantes avanzados, para los cuales se exige supervisión. Recientemente, la Sociedad Internacional de Hipnosis (ISH) ratificó entre sus cláusulas que solo la/os profesionales de la salud pueden utilizar la hipnosis con fines clínicos e investigativos (ISH, 2011). Es más fácil entender esta postura si reconocemos que la hipnosis no es un tratamiento en sí mismo y que éste se inserta en otros tratamientos para los cuales se requiere preparación profesional.

En la investigación clínica, un requerimiento para desarrollar o adaptar tratamientos es la revisión previa que documente la eficacia, necesidad 0 utilidad de las nuevas terapias (Rounsaville, Carroll, \& Onken, 2001). Los reportes de investigación en hipnosis confirman su extendido y eficaz uso en oncología. La Sociedad Americana de Cáncer y el Instituto Nacional de Cáncer de los Estados Unidos lo incluyen como un procedimiento psicológico eficaz para tratar el dolor y el estrés en el curso del tratamiento de cáncer de mama. En Puerto Rico el cáncer de mama constituye el cáncer más diagnosticado en las mujeres, sin embargo no está documentada la práctica de la hipnosis dentro de la atención psicológica a esta población. Desarrollar estrategias de intervención que integren las terapias psicológicas más eficaces resulta una exigencia para los profesionales de la psicología clínica y de la salud.

\section{CONCLUSIONES}

Esta revisión nos deja ver la rigurosidad con que ha sido asumida la hipnosis como un componente clave dentro del campo de la investigación psicosocial en pacientes de cáncer, así como su eficacia para tratar el dolor, la ansiedad anticipatoria y los efectos secundarios de los tratamientos del cáncer de mama. El consenso alcanzado en 
recientes investigaciones en cuanto a una definición y mejor descripción de los componentes de la hipnosis, contribuye a la compresión de sus resultados, elimina mitos entre pacientes y profesionales y facilita generalizar su uso.

Constituye un reto metodológico para la investigación de hipnosis en cáncer de mama diseñar investigaciones con muestras más grandes $e$ intervenciones con seguimiento a largo plazo. Desde el punto de vista sociodemógrafico, se requerirá diversificar las muestras para incluir mujeres de escasos recursos y de diversos grupos étnicos 0 raciales. La diseminación de estas intervenciones supone la traducción y adaptación de instrumentos y manuales de intervención para la población latina en Estados Unidos y de los países latinoamericanos con una alta prevalencia de cáncer de mama. La capacitación o adiestramiento en procedimientos psicológicos con abundante evidencia de eficacia clínica e investigativa como la hipnosis, debe ser visto como una responsabilidad ética por los grupos profesionales y escuelas de formación en psicología clínica.

De esta revisión se desprende la utilidad de la sugestión hipnótica para tratar síntomas de ansiedad, dolor, depresión y sofocos en pacientes de cáncer durante la trayectoria del cáncer de mama. También la hipnosis podría contribuir a mejorar dificultades con el sueño, sufrimiento emocional, fatiga y mejorar la calidad de vida de las personas con cáncer.

Aunque la hipnosis utilizada como único procedimiento ha mostrado ser eficaz para disminuir los sofocos y el estrés de la cirugía de mama, la combinación de Hipnosis con Terapia Cognitiva Conductual se consolida como una alternativa viable para tratar efectos secundarios del diagnóstico y el tratamiento del cáncer mama. Para la práctica médica y psicológica los resultados de la eficacia de la hipnosis en pacientes de cáncer sientan las bases para incluir este procedimiento como parte del cuidado estándar en los centros de oncología. La investigación en este campo de la hipnosis aún tiene obstáculos que superar pero sin dudas ha contribuido a que las pacientes diagnosticadas con cáncer identifiquen en la hipnosis un recurso valioso para mejorar su estado de ánimo y calidad de vida. Discutir los resultados y asuntos metodológicos de los trabajos de hipnosis en el cáncer de mama, confirma que una fórmula efectiva para desterrar mitos, dudas y preocupaciones sobre su uso, es investigar, publicar y practicar. 
TABLA 1.

Descripción de estudios de hipnosis y cáncer de mama. (Parte 1)

\begin{tabular}{|c|c|c|c|c|c|c|}
\hline Autores y año & $\begin{array}{c}\text { Total de } \\
\text { participantes }\end{array}$ & Edades & $\begin{array}{l}\text { Momento de la } \\
\text { intervención }\end{array}$ & Diseño & Instrumentos & Resultados \\
\hline $\begin{array}{l}\text { Spiegel y Bloom } \\
1983 . \\
\end{array}$ & $\begin{array}{l}54 \text { pacientes con } \\
\text { carcinoma } \\
\text { metástico de } \\
\text { mama. }\end{array}$ & $\begin{array}{l}\text { Promedio de 54- } \\
55 \text { años }\end{array}$ & $\begin{array}{l}\text { En tratamiento de } \\
\text { quimioterapia. }\end{array}$ & $\begin{array}{l}\text { Ensayo clínico } \\
\text { aleatorizado. }\end{array}$ & $\begin{array}{l}\text { Escala de Rango } \\
\text { del Dolor y } \\
\text { POMS. }\end{array}$ & $\begin{array}{l}\text { Ambos grupos reportaron significativamente } \\
\text { sentir menos dolor sufrimiento que la muestra } \\
\text { control. Los que recibieron terapia de grupo y } \\
\text { entrenamiento en auto-hipnosis controlaron } \\
\text { mejor la sensación de dolor. La frecuencia y } \\
\text { duración del dolor no se vieron afectadas. }\end{array}$ \\
\hline Steggles, 1999 & $\begin{array}{l}2 \text { pacientes de } \\
\text { cáncer de mama. }\end{array}$ & 54 y 58 años & $\begin{array}{l}\text { Tres semanas } \\
\text { previo a la } \\
\text { radioterapia. }\end{array}$ & Estudio de caso. & $\begin{array}{l}\text { Escala de } \\
\text { Unidades } \\
\text { subjetivas de } \\
\text { angustia (SUDS). }\end{array}$ & $\begin{array}{l}\text { Una paciente demostró disminución } \\
\text { significativa en la ansiedad. Mientras que la } \\
\text { otra paciente, terminó con unos niveles } \\
\text { menores de ansiedad en comparación al } \\
\text { principio de la intervención, }\end{array}$ \\
\hline Marchioro et.al 2000.. & $\begin{array}{l}16 \text { adultos con } \\
\text { cáncer y } 5 \text { tenían } \\
\text { cáncer de mama. }\end{array}$ & $\begin{array}{l}\text { Promedio de } 44 \\
\text { años } \\
\text { Entre 19-65 } \\
\text { años }\end{array}$ & $\begin{array}{l}\text { Durante } 4 \text { ciclos } \\
\text { de quimioterapia. }\end{array}$ & $\begin{array}{l}\text { Ensayo clínico } \\
\text { no aleatorizado. }\end{array}$ & & $\begin{array}{l}\text { Las nauseas anticipatorias y los vómitos } \\
\text { disminuyeron en todos los participantes. } \\
\text { Respuestas mayores en el control de la } \\
\text { emesis inducida por la quimioterapia fueron } \\
\text { identificadas en casi todos los pacientes. }\end{array}$ \\
\hline
\end{tabular}


TABLA 1.

Descripción de estudios de hipnosis y cáncer de mama. (Parte 2)

\begin{tabular}{|c|c|c|c|c|c|c|}
\hline Autores y año & $\begin{array}{c}\text { Total de } \\
\text { participantes }\end{array}$ & Edades & $\begin{array}{c}\text { Momento de la } \\
\text { intervención }\end{array}$ & Diseño & Instrumentos & Resultados \\
\hline Montgomery et.al 2002. & $\begin{array}{l}20 \text { pacientes de } \\
\text { biopsia de mama } \\
\text { por extracción. }\end{array}$ & $\begin{array}{l}\text { Promedio } \\
50.11 \text { años } \\
30-81 \text { años. }\end{array}$ & Pre cirugía. & $\begin{array}{l}\text { Ensayo clínico } \\
\text { aleatorizado. }\end{array}$ & $\begin{array}{l}\text { Escala Visual } \\
\text { Análoga (VAS). }\end{array}$ & $\begin{array}{l}\text { Los pacientes en el grupo } \\
\text { de hipnosis se } \\
\text { beneficiaron más que los } \\
\text { del grupo control. } \\
\text { Demostraron menos } \\
\text { dolor post quirúrgico y } \\
\text { angustia. }\end{array}$ \\
\hline Elkins et.al 2007. & $\begin{array}{l}16 \text { pacientes de } \\
\text { cáncer de mama. }\end{array}$ & $\begin{array}{l}\text { Promedio } \\
\text { 53.7.años }\end{array}$ & $\begin{array}{l}\text { Postratamiento de } \\
\text { quimioterapia }\end{array}$ & $\begin{array}{l}\text { Ensayo clínico no } \\
\text { aleatorizado. }\end{array}$ & $\begin{array}{l}\text { Escala Diaria de } \\
\text { Sofocos y Escala } \\
\text { de interferencias } \\
\text { diarias relativas a } \\
\text { sofocos }\end{array}$ & $\begin{array}{l}\text { Se mostró un } 59 \% \text { de } \\
\text { disminución en el total de } \\
\text { los hot flashes diarios y } \\
\text { un } 70 \% \text { en los } \\
\text { semanales. } \\
\text { Disminución significativa } \\
\text { en la manera en que los } \\
\text { hot flashes intervenían en } \\
\text { sus actividades diarias. }\end{array}$ \\
\hline Montgomery et.al 2007. & $\begin{array}{l}200 \text { pacientes de } \\
\text { biopsia de mama } \\
\text { por extracción. }\end{array}$ & $\begin{array}{l}\text { Promedio } \\
\text { de } 48.5 \\
\text { años. }\end{array}$ & Pre cirugía. & $\begin{array}{l}\text { Ensayo clínico } \\
\text { aleatorizado. }\end{array}$ & $\begin{array}{l}\text { Escala Visual } \\
\text { Análoga (VAS). }\end{array}$ & $\begin{array}{l}\text { Pacientes en el grupo de } \\
\text { hipnosis necesitaron } \\
\text { menos analgésicos } \\
\text { (propofol y lidocaine) que } \\
\text { los del grupo control. } \\
\text { También reportaron } \\
\text { menos dolor, } \\
\text { incomodidad, nausea, } \\
\text { fatiga, malestar y } \\
\text { disgusto emocional. }\end{array}$ \\
\hline
\end{tabular}


TABLA 1.

Descripción de estudios de hipnosis y cáncer de mama. (Parte 3)

\begin{tabular}{|c|c|c|c|c|c|c|}
\hline Autores y año & $\begin{array}{c}\text { Total de } \\
\text { participantes }\end{array}$ & Edades & $\begin{array}{l}\text { Momento de la } \\
\text { intervención }\end{array}$ & Diseño & Instrumentos & Resultados \\
\hline Elkins et.al 2008. & $\begin{array}{l}60 \text { pacientes con } \\
\text { historial de cáncer } \\
\text { de mama y } 14 \text { hot } \\
\text { flashes } 2 \text { por } \\
\text { semana. }\end{array}$ & $\begin{array}{l}18 \text { años en } \\
\text { adelante. }\end{array}$ & $\begin{array}{l}\text { Postratamiento de } \\
\text { quimioterapia }\end{array}$ & $\begin{array}{l}\text { Ensayo clínico } \\
\text { aleatorizado. }\end{array}$ & $\begin{array}{l}\text { Escala Diaria de } \\
\text { Sofocos y Escala } \\
\text { de interferencias } \\
\text { diarias relativas a } \\
\text { sofocos. } \\
\text { Escala de } \\
\text { Depresión del } \\
\text { Centro de Estudios } \\
\text { Epidemiológicos, } \\
\text { Escala Hospitalaria } \\
\text { de Depresión y } \\
\text { Escala de estudios } \\
\text { médicos de sueño }\end{array}$ & $\begin{array}{l}\text { Los hot flashes disminuyeron un } 68 \% \text {. } \\
\text { Se demostraron mejoras significativas en } \\
\text { la ansiedad, depresión, interferencia en la } \\
\text { vida diaria y el sueño, para los pacientes } \\
\text { que recibieron hipnosis. }\end{array}$ \\
\hline $\begin{array}{l}\text { Schnur \& Montgomery } \\
2008 .\end{array}$ & $\begin{array}{l}2 \text { pacientes } \\
\text { cáncer de mama } \\
\text { y radioterapia. }\end{array}$ & $\begin{array}{l}\text { Aproximadamente } \\
50 \text { años. }\end{array}$ & $\begin{array}{l}\text { Operadas de } \\
\text { cáncer de mama y } \\
\text { por comenzar } \\
\text { radioterapia. }\end{array}$ & Estudio de caso. & $\begin{array}{l}\text { Escala Visual } \\
\text { Análoga (VAS). } \\
\text { (100mm).Subescala } \\
\text { de Fatiga de la } \\
\text { Evaluación } \\
\text { funcional de la de la } \\
\text { Terapia de } \\
\text { Enfermedades } \\
\text { Crónicas. }\end{array}$ & $\begin{array}{l}\text { Aunque ambos pacientes mostraron un } \\
\text { aumento de fatiga, angustia e incomodidad } \\
\text { (en el VAS) } \\
\text { Los niveles del participante recibiendo } \\
\text { CBTH estuvieron más bajos y en control } \\
\text { que el participante en la ayuda clínica } \\
\text { estándar. }\end{array}$ \\
\hline
\end{tabular}


TABLA 1.

Descripción de estudios de hipnosis y cáncer de mama. (Parte 4)

\begin{tabular}{|c|c|c|c|c|c|c|}
\hline Autores y año & $\begin{array}{c}\text { Total de } \\
\text { participantes }\end{array}$ & Edades & $\begin{array}{l}\text { Momento de la } \\
\text { intervención }\end{array}$ & Diseño & Instrumentos & Resultados \\
\hline Schnur et.al 2009. & $\begin{array}{l}40 \text { pacientes de } \\
\text { cáncer de mama. }\end{array}$ & $\begin{array}{l}\text { 30-80 años de } \\
\text { edad. }\end{array}$ & $\begin{array}{l}\text { Recibiendo } \\
\text { tratamiento de } \\
\text { radioterapia. }\end{array}$ & $\begin{array}{l}\text { Ensayo clínico } \\
\text { aleatorizado. }\end{array}$ & $\begin{array}{l}\text { Subescala de } \\
\text { Neuroticismo del } \\
\text { Inventario de los } \\
\text { cinco Factores } \\
\text { (NEO-N). } \\
\text { Inventario de } \\
\text { ansiedad rasgo } \\
\text { estado (STAI) y } \\
\text { Perfil de Estados } \\
\text { de Ánimos, versión } \\
\text { Corta (SV-POMS). }\end{array}$ & $\begin{array}{l}\text { El grupo de CBTH demostró } \\
\text { significativamente en las evaluaciones } \\
\text { semanales tener un menor afecto negativo y } \\
\text { un aumento en el afecto positivo, el cual fue } \\
\text { consecutivo y duradero. Los resultados fueron } \\
\text { significativos estadísticamente y clínicamente } \\
\text { también. }\end{array}$ \\
\hline Butler et.al 2009. & $\begin{array}{l}124 \text { pacientes } \\
\text { cáncer de mama } \\
\text { metastásico. }\end{array}$ & $\begin{array}{l}\text { Promedio de } \\
\text { 53años de edad. }\end{array}$ & $\begin{array}{l}\text { Pacientes en } \\
\text { tratamiento. }\end{array}$ & $\begin{array}{l}\text { Ensayo clínico } \\
\text { aleatorizado. }\end{array}$ & $\begin{array}{l}\text { Escala Visual } \\
\text { Análoga (VAS). }\end{array}$ & $\begin{array}{l}\text { Terapia de grupo con hipnosis redujo } \\
\text { significativamente la experiencia de dolor y } \\
\text { sufrimiento. No hubo una diferencia } \\
\text { significativa en los reportes sobre la } \\
\text { frecuencia o consistencia del dolor. } \\
\text { Participantes que eran altamente } \\
\text { hipnotizables se beneficiaron grandemente de } \\
\text { la intervención. }\end{array}$ \\
\hline $\begin{array}{l}\text { Montgomery et.al } \\
2010 .\end{array}$ & $\begin{array}{l}200 \text { pacientes } \\
\text { cáncer de mama. }\end{array}$ & $\begin{array}{l}\text { Promedio de } 48 \\
\text { años de edad. }\end{array}$ & $\begin{array}{l}\text { Durante cirugía de } \\
\text { conservación de } \\
\text { mama. }\end{array}$ & $\begin{array}{l}\text { Ensayo clínico } \\
\text { aleatorizado }\end{array}$ & $\begin{array}{l}\text { Escala Visual } \\
\text { Análoga (VAS). y } \\
\text { Perfil de Estados } \\
\text { de Ánimos, versión } \\
\text { corta(SV-POMS). }\end{array}$ & $\begin{array}{l}\text { Expectativas pre quirúrgicas y la angustia } \\
\text { deben de ser el enfoque de intervención antes } \\
\text { de una cirugía de cáncer de mama, para } \\
\text { mejorar el dolor, la nausea y la fatiga. La } \\
\text { hipnosis demostró ser efectiva y eficiente para } \\
\text { modificar ambas, y ayudó a mejorar la } \\
\text { recuperación pos quirúrgica. }\end{array}$ \\
\hline
\end{tabular}


APA. (1994). Hypnosis: a definition. Psycological hypnosis. Bulletin of Division 30, 13

Baker, F., Denniston, M., Zabora, J., Polland, A., \& Dudley, W. N. (2002). A POMS short form for cancer patients: psychometric and structural evaluation. Psychooncology, 11(4), 273-281. doi: 10.1002/pon.564

Butler, L. D., Koopman, C., Neri, E., GieseDavis, J., Palesh, O., Thorne-Yocam, K. A., et al. (2009). Effects of supportive-expressive group therapy on pain in women with metastatic breast cancer. Health Psycholology, 28(5), 579-587. doi: 2009-14439-009 [pii]10.1037/a0016124

Capafons, A. (1995). Entrevista a Irving Kirsch. Papeles del Psicólogo, 62, from www.cop.es

Carpenter, J. S. (2001). The Hot Flash Related Daily Interference Scale: A tool for assessing the impact of hot flashes on quality of life following breastcancer. Journal Pain Symptom Manage, 22, 979-989.

Costa, P. T., \& McCrae, R. R. (1992). Revised NEO personality inventory (NEO PI-R) and NEO five-factor inventory (NEO-FFI) professional manual. Odessa, FL: Psychological Assessment Resources.

Elkins, G., Jensen, M. P., \& Patterson, D. R. (2007). Hypnotherapy for the management of chronic pain. International Journal Clinical Experimental Hypnosis, 55(3), 275-287. doi:

779406774 [pii]10.1080/00207140701338621

Elkins, G., Marcus, J., Stearns, V., \& Hasan Rajab, M. (2007). Pilot evaluation of hypnosis for the treatment of hot flashes in breast cancer survivors. Psychooncology, 16(5), 487-492. doi: 10.1002/pon.1096

Elkins, G., Marcus, J., Stearns, V., Perfect, M., Rajab, M. H., Ruud, C., et al.
(2008). Randomized trial of a hypnosis intervention for treatment of hot flashes among breast cancer survivors. Journal of Clinical Oncology, 26(31), 50225026. doi: JCO.2008.16.6389 [pii]10.1200/JCO.2008.16.6389

Green, J. P., Barabasz, A. F., Barrett, D., \& Montgomery, G. H. (2005). Forging ahead: the 2003 APA Division 30 definition of hypnosis. Internationa Journal of Clinical and Experimental Hypnosis, 53(3), 259-264. doi: U8G1558G377173LQ [pii]10.1080/00207140590961321

ISH. (2011). International Society Hypnosis. Codes ethics, from http://www.ishhypnosis.org/ish-codeethics.htm

Jemal, A., Siegel, R., Ward, E., Hao, Y., Xu, J., \& Thun, M. J. (2009). Cancer statistics, 2009. Cancer Journal for Clinicians, 59(4), 225-249. doi: caac.20006 [pii] 10.3322/caac.20006

Kirsch, I., Lynn, S. J., \& Rhue, J. W. (1993). Introduction to clinical hypnosis Handbook of clinical hypnosis. (pp. 322): Washington, DC, US: American Psychological Association.

Liossi, C. (2006). Hypnosis in cancer care. Contemporary Hypnosis, 23(1), 47-57.

Marchioro, G., Azzarello, G., Viviani, F., Barbato, F., Pavanetto, M., Rosetti, F., et al. (2000). Hypnosis in the treatment of anticipatory nausea and vomiting in patients receiving cancer chemotherapy. Oncology, 59(2), 100104. doi: 12144 [pii]

Mendoza, M. E., \& Capafons, A. (2009). Eficacia de la hipnosis clínica: resumen de su evidencia empírica. Papeles del Psicólogo Retrieved 2, 30, from http://www.cop.es/papeles

Montgomery, G. H., Bovbjerg, D. H., Schnur, J. B., David, D., Goldfarb, A., Weltz, C. $R$., et al. (2007). A randomized clinical trial of a brief hypnosis intervention to control side effects in breast surgery patients. Journal of the National Cancer 
Institute, $\quad 99(17), \quad$ 1304-1312. doi: djm106 [pii]10.1093/jnci/djm106

Montgomery, G. H., David, D., Winkel, G., Silverstein, J. H., \& Bovbjerg, D. H. (2002). The effectiveness of adjunctive hypnosis with surgical patients: a metaanalysis. Anesthesia and analgesia, 94(6), 1639-1645, table of contents.

Montgomery, G. H., Hallquist, M. N., Schnur, J. B., David, D., Silverstein, J. H., \& Bovbjerg, D. H. (2010). Mediators of a brief hypnosis intervention to control side effects in breast surgery patients: response expectancies and emotional distress. Journal of Consulting and Clinical Psychology, 78(1), 80-88. doi: 2010-00910-012 [pii]10.1037/a0017392

Montgomery, G. H., \& Schnur, J. B. (2005). Eficacia y aplicación de la hipnosis clínica. Papeles del Psicólogo, 25(089), 3-8.

Montgomery, G. H., Weltz, C. R., Seltz, M., \& Bovbjerg, D. H. (2002). Brief presurgery hypnosis reduces distress and pain in excisional breast biopsy patients. International Journal of Clinical and Experimental Hypnosis, 50(1), 17-32. doi: $10.1080 / 00207140208410088$

Redd, W. H., \& Andrykowski, M. A. (1982). Behavioral intervention in cancer treatment: controlling aversion reactions to chemotherapy. Journal of Consulting and Clinical Psychology, 50(6), 1018-1029. doi: 10.1037/0022006x.50.6.1018

Registro Central de Cáncer de Puerto Rico. (2008). El cáncer de mama en Puerto Rico. Boletín del Registro de Cáncer de Puerto Rico, 4(1). Retrieved from http://www.salud.gov.pr

Richardson, J., Smith, J. E., McCall, G., Richardson, A., Pilkington, K., \& Kirsch, I. (2007). Hypnosis for nausea and vomiting in cancer chemotherapy: a systematic review of the research evidence. European Journal of Cancer Care, 16(5), 402-412. doi: 10.1111/j.1365-2354.2006.00736.x
Rounsaville, B. J., Carroll, K. M., \& Onken, L. S. (2001). A stage model a behavioral therapies research: Getting started and moving on from stage I. Clinical Psychology: Science and Practice, 8(2), 133-142.

Schnur, J. B., David, D., Kangas, M., Green, S., Bovbjerg, D. H., \& Montgomery, G. H. (2009). A randomized trial of a cognitive-behavioral therapy and hypnosis intervention on positive and negative affect during breast cancer radiotherapy. Journal of Clinical Psychology, 65(4), 443-455. doi: 10.1002/jclp.20559

Schnur, J. B., \& Montgomery, G. H. (2008). Hypnosis and cognitive-behavioral therapy during breast cancer radiotherapy: a case report. American Journal of Clinical Hypnosis, 50(3), 209-215.

Shacham, S. (1983). A shortened version of the profile of mood states. Journal of Personality Assessment, 47(3), 305306. doi: 10.1207/s15327752jpa4703_14

Spiegel, D., \& Bloom, J. R. (1983). Group therapy and hypnosis reduce metastatic breast carcinoma pain. Psychosomatic Medicine, 45(4), 333339.

Spielberger, C. D. (1983). Manual for the state-trait anxiety inventory. Palo Alto, CA: Consulting PsychologistsPress, Inc.

Steggles, S. (1999). The use of cognitivebehavioral treatment including hypnosis for claustrophobia in cancer patients. American Journal of Clinical Hypnosis, 41(4), 319-326.

Stewart, A. L., \& Ware, J. E. (1992). Measuring functioning and well-being: The medical outcomes study approach. Durham, NC: Duke University Press

Zigmond, A. S., \& Snaith, R. P. (1983). The hospital anxiety and depression scale. Acta Psychiatrica Scandinavica, 67(6), 361-370. 